PROCEEDINGS OF THE

AMERICAN MATHEMATICAL SOCIETY

Volume 129, Number 9, Pages 2783-2788

S 0002-9939(01)05852-X

Article electronically published on January 18, 2001

\title{
SHAPE ASPHERICAL COMPACTA-APPLICATIONS OF A THEOREM OF KAN AND THURSTON TO COHOMOLOGICAL DIMENSION AND SHAPE THEORIES
}

\author{
TAKAHISA MIYATA
}

(Communicated by Ronald A. Fintushel)

\begin{abstract}
Dydak and Yokoi introduced the notion of shape aspherical compactum. In this paper, we use this notion to obtain a generalization of Kan and Thurston theorem for compacta and pro-homology. As an application, we obtain a characterization of cohomological dimension with coefficients in $\mathbb{Z}$ and $\mathbb{Z} / p$ ( $p$ prime) in terms of acyclic maps from a shape aspherical compactum, which improves the theorems of Edwards and Dranishnikov. Furthermore, we obtain the shape version of the theorem and as a consequence we show that every compactum has the stable shape type of a shape aspherical compactum.
\end{abstract}

\section{INTRODUCTION}

First recall

Theorem 1.1 (Kan and Thurston [KT]). For each path-connected space $X$, there exist a space $T X$ and a map $t: T X \rightarrow X$, natural for maps on $X$, with the following properties:

(KT1): $t_{*}: H_{*}\left(T X ; t^{*} A\right) \rightarrow H_{*}(X ; A)$ and $t^{*}: H^{*}(X ; A) \rightarrow H^{*}\left(T X ; t^{*} A\right)$ are isomorphisms of singular homologies and cohomologies with local coefficients; and

(KT2): $t_{*}: \pi_{1}(T X) \rightarrow \pi_{1}(X)$ is onto, and $\pi_{i}(T X) \cong 0$ for $i \neq 1$.

Maunder gave a simpler proof to the theorem and obtained the following variation:

Theorem 1.2 (Maunder [Ma]). For each finite connected simplicial complex $K$, there exist a finite simplicial complex $T K$ of the same dimension, and a map $t_{K}$ : $T K \rightarrow K$, natural for simplicial maps on $K$, with properties (KT1) and (KT2).

Throughout the paper, a compactum means a compact metric space, and a continuum means a connected compactum.

The paper consists of three parts. In the first part, we generalize those results as follows:

Received by the editors August 16, 1999 and, in revised form, December 29, 1999.

1991 Mathematics Subject Classification. Primary 55M10, 55P55; Secondary 54F45, 55 N05.

Key words and phrases. Shape aspherical compactum, approximately aspherical compactum, cohomological dimension, shape, Kan and Thurston theorem. 
Theorem A. For each continuum $X$ (resp., continuum with $\operatorname{dim} X<\infty$ ), there exist an approximately aspherical compactum $Y$ (resp., approximately aspherical compactum $Y$ with $\operatorname{dim} Y=\operatorname{dim} X)$ and a surjective map $\varphi: Y \rightarrow X$ with the following properties:

(S1): $\varphi$ induces isomorphisms of Čech homologies and cohomologies;

(S2): $\varphi_{*}: \operatorname{pro}-\pi_{1}^{S}(Y) \rightarrow$ pro- $\pi_{1}^{S}(X)$ is an epimorphism; and

(S3): For each connected closed subset $A$ of $X, \varphi^{-1}(A)$ is an aproximately aspherical compactum, and $\varphi \mid \varphi^{-1}(A): \varphi^{-1}(A) \rightarrow A$ satisfies properties (S1) and (S2).

Here, for each compactum $X, \operatorname{dim} X$ denotes the covering dimension of $X$. A compactum $X$ is said to be approximately aspherical if every map of $X$ into a polyhedron factors up to homotopy through a finite aspherical CW complex. Note that our definition is slightly stronger than the original definition of shape asphericity of Dydak and Yokoi [DY] by requiring the finiteness of the factoring CW complex. Asphericity of compacta in the study of cell-like maps was first considered by Daverman [Da and continued by Daverman and Dranishnikov [DD].

As an application of Theorem A, in the second part of the paper we obtain a characterization of cohomological dimension with coefficients in $\mathbb{Z}$ and $\mathbb{Z} / p$ for any prime $p$, which improves the well-known characterizations by Edwards and Dranishnikov in the theorems below.

For each compactum $X$ and abelian group $G$, the cohomological dimension $\operatorname{cdim}_{G} X \leq n$ if $X \tau K(G, n)$, where for any ANR $P, X \tau P$ denotes the property that every map of any closed subset of $X$ into $P$ extends over $X$.

Theorem 1.3 (Edwards [E,, $\mathrm{W}]$ ). For each compactum $X, \operatorname{cdim}_{\mathbb{Z}} X \leq n$ if and only if there exists a cell-like map $f: Y \rightarrow X$ from a compactum $Y$ of $\operatorname{dim} Y \leq n$.

Theorem 1.4 (Dranishnikov Dr). For each compactum $X$ and for each prime $p$, $\operatorname{cdim}_{\mathbb{Z} / p} X \leq n$ if and only if there exists a surjective map $f: Y \rightarrow X$ from a compactum $Y$ of $\operatorname{dim} Y \leq n$ such that each fibre is acyclic modulo $p$.

Koyama $[\mathrm{K}]$ and Koyama and Yokoi $[\mathrm{KY}]$ extended those results to approximable dimensions with arbitrary coefficient groups. Note the approximable dimension with a finitely generated coefficient group coincides with the cohomological dimension.

We obtain the following:

Theorem B. For each continuum $X$ and for each prime $p, \operatorname{cdim}_{\mathbb{Z}} X \leq n$ (resp., $\operatorname{cdim}_{\mathbb{Z} / p} X \leq n$ ) if and only if there exist an approximately aspherical compactum $Y$ with $\operatorname{dim} Y \leq n$ and a surjective map $f: Y \rightarrow X$ such that each fibre is acyclic (resp., acyclic modulo $p$ ).

In the third and final part of the paper we give applications to shape theory. Let sd $X$ denote the shape dimension of $X$ (see MaS, p. 95]).

Theorem C. For each continuum $X$ of $\mathrm{sd} X<\infty$, there exist an approximately aspherical compactum $Y$ of $\operatorname{dim} Y=\operatorname{sd} X$ and a shape morphism $\varphi: Y \rightarrow X$ with properties (S1) and (S2).

Theorem D. $\quad$ 1. Every continuum has the weak stable shape type of an approximately aspherical compactum. 
2. Every continuum $X$ of $\operatorname{sd} X<\infty$ has the stable shape type of an approximately aspherical compactum $Y$ of $\operatorname{dim} Y=\operatorname{sd} X$.

See MiS1 for the definitions in stable shape theory.

\section{Characterizations of APPRoXimately aspherical COMPACTA}

Theorem 2.1. For every compactum $X$, the following are equivalent:

i) $X$ is an approximately aspherical compactum;

ii) $X$ admits an expansion of $X, \mathbf{p}=\left(p_{i}\right): X \rightarrow \mathbf{X}=\left(X_{i}, p_{i i+1}, \mathbb{N}\right)$, such that each $X_{i}$ is a finite aspherical polyhedron (here, the expansion is in the sense of [MaS, p. 19]); and

iii) Every polyhedral expansion of $X, \mathbf{p}=\left(p_{i}\right): X \rightarrow \mathbf{X}=\left(X_{i}, p_{i i+1}, \mathbb{N}\right)$ has the property that every $i$ admits $i^{\prime} \geq i$ such that $p_{i i^{\prime}}$ factors through a finite aspherical polyhedron.

Proof. ii) $\Rightarrow$ i) is obvious. We wish to verify i) $\Rightarrow$ iii) $\Rightarrow$ ii). For i) $\Rightarrow$ iii), let $\mathbf{p}=\left(p_{i}\right): X \rightarrow \mathbf{X}=\left(X_{i}, p_{i i+1}, \mathbb{N}\right)$ be a polyhedral expansion of $X$. For each $i$, there exist a finite aspherical polyhedron $P$ and homotopy maps $g: X \rightarrow P$ and $h: P \rightarrow X_{i}$ such that $p_{i}=h g$. Then for some $i^{\prime \prime} \geq i$ there exists a homotopy map $g^{\prime}: X_{i^{\prime \prime}} \rightarrow P$ such that $g=g^{\prime} p_{i^{\prime \prime}}$. So, $p_{i i^{\prime \prime}} p_{i^{\prime \prime}}=p_{i}=h g^{\prime} g_{i^{\prime \prime}}$, and hence there exists $i^{\prime} \geq i^{\prime \prime}$ such that $p_{i i^{\prime}}=h g^{\prime} p_{i^{\prime \prime} i^{\prime}}$ as desired. For iii) $\Rightarrow$ ii), let $\mathbf{p}=\left(p_{i}\right): X \rightarrow$ $\mathbf{X}=\left(X_{i}, p_{i i+1}, \mathbb{N}\right)$ be any polyhedral expansion of $X$. Then by iii), there is an increasing sequence $1=i_{1} \leq i_{2} \leq \cdots \leq i_{k} \leq \cdots$ and finite aspherical polyhedra $Y_{k}$, $k=1,2, \ldots$, such that $p_{i_{k} i_{k+1}}=h_{k} g_{k}$ for some $g_{k}: X_{i_{k+1}} \rightarrow Y_{k}$ and $h_{k}: Y_{k} \rightarrow X_{i_{k}}$. For each $k=1,2, \ldots$, let $q_{k}=g_{k} p_{i_{k+1}}: X \rightarrow Y_{k}$ and $q_{k k+1}=g_{k} h_{k+1}: Y_{k+1} \rightarrow Y_{k}$. Then it is a routine to check that $\mathbf{q}=\left(q_{k}\right): X \rightarrow \mathbf{Y}=\left(Y_{k}, q_{k k+1}, \mathbb{N}\right)$ forms an expansion of $X$.

Remark 2.2. Analogous characterization for Dydak and Yokoi's definition holds without the finiteness conditions on the aspherical polyhedra in ii) and iii).

\section{Proof of Theorem A}

Before we prove the theorem, we observe the following properties for the map $t_{K}: T K \rightarrow K$ in Maunder's Theorem, which were obtained in the original proof Ma:

(M1): For each connected subcomplex $M$ of $K, \operatorname{dim} t_{K}^{-1}(M)=\operatorname{dim} M$, and $t_{K} \mid t_{K}^{-1}(M): t_{K}^{-1}(M) \rightarrow M$ satisfies properties (KT1) and (KT2) and is natural in the following sense: For any simplicial map $f: K \rightarrow K^{\prime}$, if $L$ and $L^{\prime}$ are subcomplexes of $K$ and $K^{\prime}$, respectively, such that $f(L) \subseteq L^{\prime}$, then the following diagram commutes:

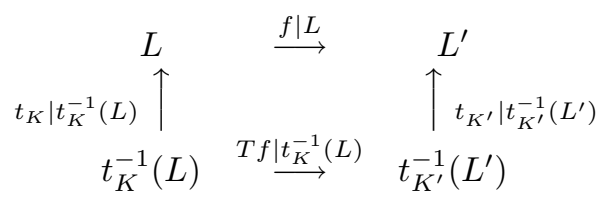

where $T f: T K \rightarrow T K^{\prime}$ is the induced simplicial map;

(M2): Each fibre of $t_{K}$ is either a point or an acyclic and aspherical subcomplex of $T K$; and

(M3): $t_{K}$ is onto. 
Let $X$ be a continuum, and let $\mathfrak{U}_{i}, i=1,2, \ldots$, be a sequence of finite open coverings of $X$ which form a base for the toplogy on $X$. For each $i$, let $K_{i}$ be the nerve of $\mathfrak{U}_{i}$ with realization $X_{i}$, let $p_{i i+1}: K_{i+1} \rightarrow K_{i}$ be a connecting simplicial map and let $p_{i}: X \rightarrow X_{i}$ be a canonical map. Then the map $\mathbf{p}=\left(p_{i}\right): X \rightarrow \mathbf{X}=$ $\left(X_{i}, p_{i i+1}, \mathbb{N}\right)$ forms an inverse limit. By Theorem 1.2 and the above observation, for each $i$, there exist a complex $T K_{i}$ and a map $\varphi_{i}=t_{K_{i}}: T K_{i} \rightarrow K_{i}$ with properties (KT1), (KT2), (M1), (M2) and (M3) and a simplicial map $q_{i i+1}=$ $T p_{i i+1}: T K_{i+1} \rightarrow T K_{i}$ which makes the following diagram commute:

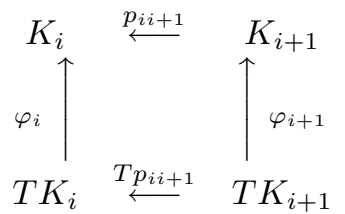

For each $i$, let $Y_{i}$ be the realization of $T K_{i}$, and let $Y$ be the limit of the inverse sequence $\mathbf{Y}=\left(Y_{i}, q_{i i+1}, \mathbb{N}\right)$ with the projections $q_{i}: Y \rightarrow Y_{i}$. Then the level morphism $\varphi=\left(\varphi_{i}\right): \mathbf{Y} \rightarrow \mathbf{X}$ induces the limit map $\varphi: Y \rightarrow X$, which is surjective. By properties (KT1) and (KT2) for each $\varphi_{i}: Y_{i} \rightarrow X_{i}, \varphi$ satisfies properties (S1) and (S2). To verify property (S3), let $A$ be a closed subset of $X$. For each $i$, let $L_{i}$ be the nerve of the open covering $\mathfrak{U}_{i} \mid A=\left\{U \cap A: U \in \mathfrak{U}_{i}\right\}$ of $A$. Then $L_{i}$ is a subcomplex of $K_{i}$. So, for each $i$, if $A_{i}$ is the realization of $L_{i}$, then $A_{i}$ is a subpolyhedron of $X_{i}$, and by property (M1) we have the following commutative diagram:

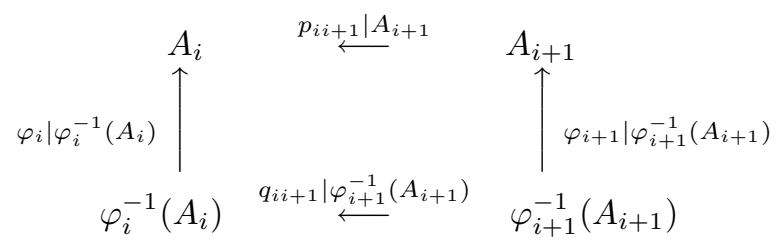

and each $\varphi_{i} \mid \varphi_{i}^{-1}\left(A_{i}\right): \varphi_{i}^{-1}\left(A_{i}\right) \rightarrow A_{i}$ satisfies properties (KT1) and (KT2). Note that the restricted maps $\mathbf{p} \mid A=\left(p_{i} \mid A\right): A \rightarrow \mathbf{A}=\left(A_{i}, p_{i i+1} \mid A_{i}, \mathbb{N}\right)$ and $\mathbf{q} \mid \varphi^{-1}(A)=$ $\left(\mathbf{q}_{i} \mid \varphi^{-1}(A)\right): \varphi^{-1}(A) \rightarrow \varphi^{-1}(\mathbf{A})=\left(\varphi^{-1}\left(A_{i}\right), \mathbf{q}_{i i+1} \mid \varphi_{i+1}^{-1}\left(A_{i+1}\right), \mathbb{N}\right)$ form the inverse limits of $A$ and $\varphi^{-1}(A)$, respectively. So the map $\varphi \mid \varphi^{-1}(A): \varphi^{-1}(A) \rightarrow A$ which is the limit map of the level morphism $\varphi \mid \varphi^{-1}(\mathbf{A})=\left(\varphi_{i} \mid \varphi_{i}^{-1}\left(A_{i}\right)\right): \varphi^{-1}(\mathbf{A}) \rightarrow$ A has properties (S1) and (S2). Since each $\varphi_{i}^{-1}\left(A_{i}\right)$ is aspherical, $\varphi^{-1}(A)$ is approximately aspherical by Proposition 2.1 Hence property (S3) is fulfilled.

Now suppose $\operatorname{dim} X=n<\infty$. Then we can take the base $\mathfrak{U}_{i}, i=1,2, \ldots$, so that the nerves of $\mathfrak{U}_{i}$ have dimension at most $n$. So, for each $i, \operatorname{dim} Y_{i}=\operatorname{dim} T K_{i}=$ $\operatorname{dim} K_{i} \leq n$, and hence $\operatorname{dim} Y \leq n$. On the other hand, the commutative diagram for each closed subset $A$ of $X$

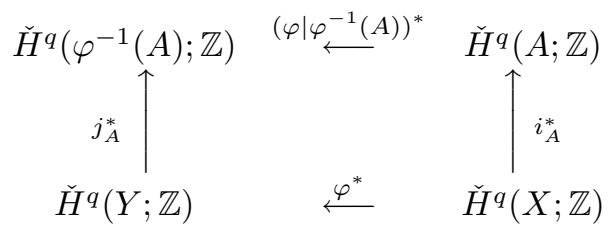

and property (S3) imply $\operatorname{cdim}_{\mathbb{Z}} X \leq \operatorname{cdim}_{\mathbb{Z}} Y$, and by Alexandroff theorem, $\operatorname{dim} X=$ $\operatorname{cdim}_{\mathbb{Z}} X$ and $\operatorname{cdim}_{\mathbb{Z}} Y=\operatorname{dim} Y$. Hence $\operatorname{dim} Y=n$, as required. 


\section{Proof of Theorem B}

Assume there is a surjective map $f: Y \rightarrow X$ from an approximately aspherical compactum $Y$ with $\operatorname{dim} Y \leq n$ such that $\check{H}^{*}\left(f^{-1}(x) ; \mathbb{Z}\right)=0$ for all $x \in X$. Using Vietoris-Begle theorem, we can obtain $\operatorname{cdim}_{\mathbb{Z}} X \leq \operatorname{cdim}_{\mathbb{Z}} Y=\operatorname{dim} Y \leq n$. Hence $\operatorname{cdim}_{\mathbb{Z}} X \leq n$.

Conversely, suppose $\operatorname{cdim}_{\mathbb{Z}} X \leq n$. Then Edwards theorem (Theorem 1.3]) implies that there exists a cell-like map $g: X^{\prime} \rightarrow X$ from a compactum $X^{\prime}$ with $\operatorname{dim} X^{\prime} \leq n$. By taking each component of $X^{\prime}$, without loss we can assume $X^{\prime}$ is connected. Theorem A implies that there exists a surjective map $h: Y \rightarrow X^{\prime}$ from a shape aspherical map $Y$ of $\operatorname{dim} Y=\operatorname{dim} X^{\prime}$ such that for each closed subset $B$ of $X^{\prime}$, the restricted map $h \mid h^{-1}(B): h^{-1}(B) \rightarrow B$ induces an isomorphism $\left(h \mid h^{-1}(B)\right)^{*}: \check{H}^{q}(B ; \mathbb{Z}) \rightarrow \check{H}^{q}\left(h^{-1}(B) ; \mathbb{Z}\right)$ for each $q$. So, if we let $f=g h: Y \rightarrow X$, then $\check{H}^{q}\left(f^{-1}(x) ; \mathbb{Z}\right) \cong \check{H}^{q}\left(g^{-1}(x) ; \mathbb{Z}\right) \cong 0$ for each $q$. The case for $\mathbb{Z} / p$ is proved similarly, using Dranishnikov theorem (Theorem 1.4).

\section{Proofs of Theorems C And D}

Proof of Theorem C. If $\operatorname{sd} X \leq n<\infty$, then there is a polyhedral expansion $\mathbf{p}=\left(p_{i}\right): X \rightarrow \mathbf{X}=\left(X_{i}, p_{i i+1}, \mathbb{N}\right)$ of $X$ such that $X_{i}$ are finite polyhedra with $\operatorname{dim} X_{i} \leq n$. Then choose a triangulation $K_{1}$ of $X_{1}$, and using the simplicial approximation theorem, we can inductively choose triangulations $K_{i}$ of $X_{i}$ and simplicial maps $a_{i i+1}: K_{i+1} \rightarrow K_{i}$ that represent the corresponding homotopy classes $p_{i+1}: X_{i+1} \rightarrow X_{i}$. As in the proof of Theorem A, for each $i$, there exist a simplicial complex $T K_{i}$ with $\operatorname{dim} T K_{i}=\operatorname{dim} K_{i}$ and maps $\varphi_{i}: T K_{i} \rightarrow K_{i}$ with properties (KT1) and (KT2) and $q_{i i+1}: T K_{i+1} \rightarrow T K_{i}$. Let $Y$ be the limit of the inverse sequence $\mathbf{Y}=\left(Y_{i}, q_{i i+1}, \mathbb{N}\right)$ where $Y_{i}$ are the realizations of $T K_{i}$, and let $q_{i}: Y \rightarrow Y_{i}$ be the projection maps. Then $\mathbf{q}=\left(q_{i}\right): Y \rightarrow \mathbf{Y}$ induces a polyhedral expansion of $Y$, so the maps $\varphi_{i}: Y_{i} \rightarrow X_{i}$ form a level morphism $\varphi=\left(\varphi_{i}\right): \mathbf{Y} \rightarrow \mathbf{X}$ which represents a shape morphism $\varphi: Y \rightarrow X$ with properties (S1) and (S2). Since $\operatorname{dim} Y_{i}=\operatorname{dim} X_{i} \leq n$, then $\operatorname{dim} Y \leq n$.

Thus $\operatorname{dim} Y \leq \operatorname{sd} X$. On the other hand, by [L], property (S3) and Alexandroff Theorem, sd $X \leq \operatorname{cdim}_{\mathbb{Z}} X \leq \operatorname{cdim}_{\mathbb{Z}} Y=\operatorname{dim} Y$. Hence $\operatorname{sd} X=\operatorname{dim} Y$.

Corollary 5.1. 1. For each continuum $X$ (resp., continuum $X$ with $\operatorname{dim} X<$ $\infty)$, there exists an approximately aspherical compactum $Y$ (resp., approximately aspherical compactum $Y$ with $\operatorname{dim} Y=\operatorname{dim} X)$ and a surjective map $\varphi: Y \rightarrow X$ such that the induced map $\operatorname{SP}^{\infty}(\varphi): \mathrm{SP}^{\infty} Y \rightarrow \mathrm{SP}^{\infty} X$ is a weak shape equivalence.

2. For each continuum $X$ with $\mathrm{sd} X<\infty$, there exists an approximately aspherical compactum $Y$ with $\operatorname{dim} Y=\operatorname{sd} X$ and a shape morphism $\varphi: Y \rightarrow X$ such that the induced map $\operatorname{SP}^{\infty}(\varphi): \mathrm{SP}^{\infty} Y \rightarrow \mathrm{SP}^{\infty} X$ is a weak shape equivalence.

Proof. This easily follows from Theorems A and C and DT].

Proof of Theorem D. Let $X$ be a continuum. Then by Theorem A, there exists a map $\varphi: Y \rightarrow X$ from an approximately aspherical compactum $Y$ onto $X$ such that $\varphi_{*}: \operatorname{pro}-H_{q}(Y ; \mathbb{Z}) \rightarrow$ pro $-H_{q}(X ; \mathbb{Z})$ is an isomorphism for each $q$, which implies by [MiS2, Corollary 7.8] that $\varphi_{*}: \operatorname{pro}-\pi_{q}^{S}(Y) \rightarrow \operatorname{pro}-\pi_{q}^{S}(X)$ is an isomorphism for each $q$ as required. If $\operatorname{sd} X<\infty$, then Theorem $\mathrm{C}$ implies that there exists a shape morphism $\varphi: Y \rightarrow X$ from an approximately aspherical compactum $Y$ of 
$\operatorname{dim} Y=\operatorname{sd} X$ such that $\varphi_{*}: \operatorname{pro}-H_{q}(Y ; \mathbb{Z}) \rightarrow \operatorname{pro}-H_{q}(X ; \mathbb{Z})$ is an isomorphism for each $q$, so $\varphi_{*}:$ pro- $\pi_{q}^{S}(Y) \rightarrow$ pro- $\pi_{q}^{S}(X)$ is an isomorphism for each $q$. Now by [MiS1, Theorem 6.1], $\varphi$ is an equivalence in the stable shape category.

\section{REFERENCES}

[E] R. D. Edwards, A theorem and a question related to cohomological dimension and cell-like maps, Notices Amer. Math. Soc. 25 (1978) A-259.

[Da] R. J. Daverman, Hereditarily aspherical compacta and cell-like maps, Top. Appl. 41 (1991), 247 - 254. MR 93b:54033

[DD] R. J. Daverman and A. Dranishnikov, Cell-like maps and aspherical compacta, Illinois J. Math. 40(1) (1996), 77-90. MR 98h:54045

[DT] A. Dold and R. Thom, Quasifaserungen und unendliche symmetrische Produkte, Annals of Math. 67 (1958), 239 - 281. MR 20:3542

[Dr] A. N. Dranishnikov, On homological dimension modulo p, Math. USSR Sb. 60(2) (1988), 413 - 425. MR 88h:55001

[DY] J. Dydak and K. Yokoi, Hereditarily aspherical compacta, Proc. Amer. Math. Soc. 124 (1996), 1933 - 1940. MR 96h:57019

[KT] D. M. Kan and W. P. Thurston, Every connected space has the homology of a $K(\pi, 1)$, Topology 15 (1976), 253 - 258. MR 54:1210

[K] A. Koyama, A characterization of compacta which admit acyclic $U V^{n-1}$-resolutions, Tsukuba J. Math. 20(1) (1996), 115 - 121. MR 97d:55003

[KY] A. Koyama and K. Yokoi, A unified approach of characterizations and resolutions for cohomological dimension modulo $p$, Tsukuba J. Math. 18(2) (1994), 247-282. MR 95j:55002

[L] Yu. V. Lubenets, The fundamental dimension of subcompacta, Russian Math. Surveys 47 (1992), 182 - 183. MR 94b:54100

[MaS] S. Mardešić and J. Segal, Shape Theory, North-Holland Publishing Company, 1982. MR 84b:55020

[Ma] C. R. F. Maunder, A short proof of a theorem of Kan and Thurston, Bull. London Math. Soc. 13 (1981), 325 - 327. MR 82h:55009

[Mi] T. Miyata, Generalized stable shape and duality, Top. Appl. 109 (2001), 75 - 88.

[MiS1] T. Miyata and J. Segal, Generalized stable shape and the Whitehead theorem, Top. Appl. 63 (1995), 139 - 164. MR 96d:54017

[MiS2] T. Miyata and J. Segal, Generalized stable shape and Brown's representation theorem, Top. Appl. 94 (1999), 275 - 305. MR 2000f:54008

[W] J. J. Walsh, Dimension, cohomological dimension, and cell-like mappings, Lecture Note in Math. 870 (1981), 105 - 118. MR 83a:57021

Department of Computer Science, Shizuoka Institute of Science and Technology, 2200-2 Toyosawa, Fukuroi, Shizuoka-Pref., 437-8555 Japan

E-mail address: miyata@mb.sist.ac.jp 